\title{
Preliminary characterization of the normal microbiota of the human vulva using cultivation- independent methods
}

Correspondence

Celeste J. Brown

celesteb@uidaho.edu

Received 4 March 2006

Accepted 26 October 2006

\author{
Celeste J. Brown, ${ }^{1}$ Mayee Wong, ${ }^{1} \dagger$ Catherine C. Davis, ${ }^{2}$ Amita Kanti, ${ }^{2}$ \\ Xia Zhou ${ }^{1}$ and Larry J. Forney ${ }^{1}$ \\ ${ }^{1}$ Department of Biological Sciences, University of Idaho, Moscow, ID 83844-3051, USA \\ ${ }^{2}$ Procter \& Gamble Company, 6110 Center Hill Ave, Cincinnati, OH 45224, USA
}

\section{INTRODUCTION}

The human vulva consists of several distinct ecotopes that are defined by their physical and chemical characteristics. The physical characteristics include distinct histological architectures and anatomical proximity to the vagina, urethra, anus and Bartholin's gland, whilst the chemical factors include the composition and amount of vaginal secretions, as well as contamination by urine and faecal material. As a result, the microbiota of the human vulva is complex and unique (Elsner \& Maibach, 1990). Cultivationdependent methods have been used previously to study the microbiology of the labia majora. The microbiota of the labia majora is characterized by micro-organisms that are either related to vaginal and urethral inhabitants (such as Lactobacillus spp.) or are common for intertriginous skin, including Gram-negative rods, non-pathogenic Neisseria, Gardnerella vaginalis and Staphylococcus aureus (Aly et al., 1979; Elsner \& Maibach, 1990). Technical difficulties in the isolation of bacteria have confounded efforts to obtain a comprehensive understanding of the organisms present in various anatomical locations on the human body (Berg,

tPresent address: Center for Infectious Disease Ecology, Asia-Pacific Institute for Tropical Medicine and Infectious Diseases, 651 llalo St, BSB 320, Honolulu, HI 96813, USA.

Abbreviation: RDP II, Ribosomal Database Project.

The GenBank/EMBL/DDBJ accession numbers for the $16 \mathrm{~S}$ rRNA gene sequences determined in this study are D0975475-DQ.976361.
1999; Henderson et al., 1998; Hooper et al., 2001; Pace, 1997; Rappe \& Giovannoni, 2003; Tlaskalová-Hogenová et al., 2004). This raises the possibility that organisms that normally reside in the human vulva were overlooked in previous studies that employed cultivation-dependent methods.

Micro-organisms present on the vulva of healthy women may have clinical significance because their presence may affect the proliferation of non-indigenous populations (Atassi et al., 2006; Reid \& Burton, 2002), including those that play a crucial role in triggering various common diseases such as vulvovaginitis (Wilson, 2005), urinary tract infection (Reid, 1999), bartholinitis (Nakatsu et al., 1998; Sing et al., 1998) and abscesses of Bartholin's gland (Tanaka et al., 2005). Vulvovaginitis is characterized by inflammation or infection of the vulva and vagina. It affects women of all ages and is very common (Makela et al., 2003; Merkley, 2005; Sobel, 2001, 2003; Wilson, 2005). It can be caused by bacteria, yeasts, viruses or parasites, including some that are responsible for sexually transmitted diseases (Makela et al., 2003). Vulvovaginal candidiasis, which is caused by species of Candida, is one of the most common forms of vulvovaginitis in women of all ages (Eschenbach, 2004; Sheary \& Dayan, 2005; Sobel, 2004; Spence, 2003; Xu \& Sobel, 2004). Approximately $75 \%$ of women in the USA experience vulvovaginitis caused by Candida at some time during their reproductive years (Wilson, 2005), between 40 and $50 \%$ of women have recurrent episodes (Sheary \& 
Dayan, 2005), and 5-8\% experience chronic Candida infections (Eschenbach, 2004). As relatively little is known about the microbiology of the vulva, the aetiology and precipitating factors of vulvovaginitis are not well understood. Consequently, the treatment and management of this disease represent a large burden on the health-care system. An accurate understanding of the composition and ecology of the healthy vulvovaginal microbiota may provide insight into components of the microbiota that may reduce the risk of acquiring this and other diseases. However, to elucidate fully the potential protective effects of the healthy vulvovaginal microbiota would require comparisons between the healthy and compromised or diseased state, or between high-risk and low-risk groups.

The aim of this preliminary study was to investigate the microbial communities in the normal human vulva of four healthy adult women. Our approach utilized partial sequencing of $16 \mathrm{~S}$ rRNA genes to identify bacterial populations that were abundant in the labia majora and the labia minora.

\section{METHODS}

Clinical samples. The study protocol and informed-consent documents were reviewed and approved by the Procter \& Gamble Corporate Institutional Review Board. Five healthy white women, aged 28-44 years, were recruited at visit 1 to complete health questionnaires and provide written informed consent. During this visit, they received Olay Moisturizing Bar - Sensitive Skin (Procter \& Gamble) for body cleansing and Always Ultra Thin (Procter \& Gamble) catamenial pads, which were used for at least 1 month prior to the sampling visit in order to standardize the environment among the women. All of the women were in good general health, reported regular menstrual cycles, used the supplied cleansing bar for bathing and refrained from the following: (a) bathing $2 \mathrm{~h}$ prior to the study, (b) douching during the study and (c) sexual intercourse $48 \mathrm{~h}$ prior to the study visit. Exclusion criteria included antibiotic usage (oral or topical in the vulvar/vaginal area) for 6 weeks prior to sampling and body piercing in the vulvar, thigh or buttock area. Subjects were also excluded if they self-reported: (a) pregnancy, (b) an immunocompromised state, (c) sexually transmitted diseases, (d) AIDS and/or (e) hepatitis. Sample collection dates varied by subject, and demographic information about the participants is shown in Table 1. One subject (W1) was entered into the study but the vulvar samples did not produce a sufficient number of clones for the subsequent analyses. Participant identification coding was consistent with previous studies by Zhou et al. (2004) and Coolen et al. (2005). Separate scrape samples were collected from the labia majora and labia minora during a single visit. A health-care practitioner used separate sterile cervicovaginal sampling devices to sample a $2 \mathrm{~cm}^{2}$ area of the left labia majora and minora near the introitus. Samples were placed in separate $50 \mathrm{ml}$ sterile test tubes, stored at $-70{ }^{\circ} \mathrm{C}$ and forwarded on dry ice for molecular analyses.

Experimental procedures. Vulvar scrape samples were stored at $-20{ }^{\circ} \mathrm{C}$ and community bacterial DNA was isolated using the techniques described by Zhou et al. (2004). PCR amplification of an internal region of the $16 \mathrm{~S}$ rRNA genes from each vulvar microbial community was accomplished using the bacteria-specific primers 8Fm (5'-AGAGTTTGATCMTGGCTCAG- $\left.3^{\prime}\right)$ and 926R (5'CCGTCAATTCCTTTRAGTTT-3') (Weisburg et al., 1991). DNA template $(1 \mu \mathrm{l})$ was added to a reaction mixture containing $0.1 \mu \mathrm{M}$
Table 1. Demographics of participants in the study with analysable samples

Sample designations (W2-W5) coincide with those of Coolen et al. (2005) and Zhou et al. (2004).

\begin{tabular}{|lcccc|}
\hline Characteristic & W2 & W3 & W4 & W5 \\
\hline Age (years) & 44 & 40 & 28 & 30 \\
Race/ethnicity & White & White & White & White \\
Gravidity/parity & $3 / 3$ & $5 / 3$ & $3 / 2$ & $0 / 0$ \\
Oral contraceptive use & No & No & Yes & Yes \\
Day of menstrual cycle & 23 & 20 & 2 & 20 \\
\hline
\end{tabular}

${ }^{\star}$ Day 1 was taken as the first appearance of menses.

each primer, $0.1 \mu \mathrm{M}$ dNTPs, $200 \mu \mathrm{M} \mathrm{MgCl}_{2}, 5 \mu \mathrm{l}$ DMSO, $3 \mathrm{mM}$ GeneAmp $10 \times$ PCR Buffer II and 2 U AmpliTaq DNA polymerase (Roche Molecular Systems) in a final volume of $100 \mu$ l. Samples were denatured at $94{ }^{\circ} \mathrm{C}$ for $5 \mathrm{~min}$, followed by 36 cycles of denaturation at $94{ }^{\circ} \mathrm{C}$ for $1 \mathrm{~min}$, annealing at $55^{\circ} \mathrm{C}$ for $1 \mathrm{~min}$ and extension at $72{ }^{\circ} \mathrm{C}$ for $2 \mathrm{~min}$. The amplification step was followed by a final elongation step at $72{ }^{\circ} \mathrm{C}$ for $10 \mathrm{~min}$. All PCR amplifications used thinwalled tubes in a PTC-100 or PTC-200 thermocycler (MJ Research). Amplicons from bacterial community DNA were processed with the QIAquick PCR purification kit (Qiagen) to remove excess dinucleotides and primer dimers, and DNA concentrations were estimated by comparison with a Low DNA Mass Ladder (Invitrogen) after gel electrophoresis and ethidium bromide staining. 16S rRNA gene clone libraries were constructed using TOPO TA cloning kits (versions $\mathrm{P}$ and J; Invitrogen) using chemically competent Escherichia coli DH5 $\alpha$.

Approximately 200 colonies were picked randomly from each $16 \mathrm{~S}$ rRNA clone library. Bacterial colonies were diluted in $20 \mu \mathrm{l}$ ultrapure water and subjected to at least one freeze/thaw cycle to lyse the bacterial cells. Each lysate $(1 \mu \mathrm{l})$ was used in a $20 \mu \mathrm{l}$ amplification reaction with primers M13R (5'-CAGGAAACAGCTATGACCATGG-3') and T7 (5' TAATACGACTCACTATAGG-3'). Approximately $40-160 \mathrm{ng}$ of cloned PCR product was incubated with $1.6 \mu$ ExoSAP-IT (USB), following the manufacturer's directions, for chemical inactivation of excess dNTPs and primer dimers. Each cycle sequencing reaction contained $1 \mu \mathrm{l}$ $2.5 \mu \mathrm{M}$ M13R or T7 primer, $4 \mu \mathrm{l}$ BigDye terminator cycle sequencing ready reaction mix (version 3.0; Perkin-Elmer) and $5 \mu$ amplification product. Cycle sequencing conditions were as follows: denaturation at $95^{\circ} \mathrm{C}$ for $4 \mathrm{~min}$, followed by 26 cycles of denaturation at $96^{\circ} \mathrm{C}$ for $10 \mathrm{~s}$, annealing at $50{ }^{\circ} \mathrm{C}$ for $5 \mathrm{~s}$ and elongation at $60^{\circ} \mathrm{C}$ for $4 \mathrm{~min}$. Cycle sequence reactions were cleaned with Performa DTR 96-well short plate kits (Edge BioSystems). The DNA sequences were determined on a 3100 PRISM genetic analyser and electropherograms were analysed using the ABI PRISM AB DNA sequencing analysis software version 5.1.1 (Applied Biosystems).

Classification of clone sequences was based on genetic similarity to $16 \mathrm{~S}$ rRNA gene sequences from cultured bacteria whose 16S rRNA sequences are found in the Ribosomal Database Project (RDP II) version 8 (Cole et al., 2003). A high-throughput method was used for these analyses, such that no corrections to sequences or alignments were made. Single-strand sequences that were $>500 \mathrm{nt}$ and were in the coding direction were compared against almost full-length sequences (>1200 nt) using the BLAST algorithm (Altschul et al., 1997). For each woman, the clone sequences, the most similar sequence in RDP II for each clone and a set of one archaeal [gi no. 15668172 (159459-157985)] and 39 eubacterial sequences [Genbank accession nos: AY151243, D89032, X84812, AB045898, AJ293103, AF027653, AB062105, Y17512, 
Table 2. Phylotypes found in the clone libraries of vulvar samples from four healthy women

Each phylotype in this table was found in more than one woman or more than one location in the same woman. Data are presented as the percentage of clones examined from each sample identified as that phylotype. The median genetic divergence (base substitutions per 100 bases) of all sequences of that phylotype to the RDP II sequence of the bacterial species is given in parentheses. Sample designations (W2W5) coincide with those of Coolen et al. (2005) and Zhou et al. (2004). A, labia minora; B, labia majora; $n$, number of clones analysed.

\begin{tabular}{|c|c|c|c|c|c|c|c|c|}
\hline \multirow[t]{2}{*}{ Bacterial species ${ }^{\star}$} & \multicolumn{2}{|c|}{ W2 } & \multicolumn{2}{|c|}{ W3 } & \multicolumn{2}{|c|}{ W4 } & \multicolumn{2}{|c|}{ W5 } \\
\hline & A $(n=105)$ & B $(n=101)$ & A $(n=101)$ & B $(n=114)$ & A $(n=106)$ & B $(n=87)$ & A $(n=153)$ & B $(n=120)$ \\
\hline Lactobacillus crispatus & $70.5(0.4)$ & $14.9(0.5)$ & $97.0(0.0)$ & $22.8(0.3)$ & & & & \\
\hline Atopobium vaginae & & & & & & $1.1(0.0)$ & $26.8(0.0)$ & $17.5(0.0)$ \\
\hline Megasphaera elsdenii & & & & & & & $30.1(12.8)$ & $12.5(14.1)$ \\
\hline Enterococcus faecalis & $1.9(0.1)$ & $34.7(0.0)$ & & & & & & \\
\hline Ideonella spp. & & & & $10.5(2.7)$ & & & $0.7(3.0)$ & $2.5(3.9)$ \\
\hline Thauera aromatica & & & & $12.3(12.3)$ & & & & $0.8(11.5)$ \\
\hline Brevibacillus levickii & $12.4(5.3)$ & & & & $0.9(5.3)$ & & & \\
\hline 'Leptotrichia amnionii' & & & & & & & $7.2(0.5)$ & $2.5(0.8)$ \\
\hline Peptoniphilus lacrimalis & & & & & & & $0.7(0.5)$ & $5.0(0.5)$ \\
\hline Veillonella parvula & & $1.0(0.8)$ & & & & $1.1(0.8)$ & & \\
\hline Peptoniphilus lacrimalis & & & & & & & $0.7(8.9)$ & $0.8(8.6)$ \\
\hline
\end{tabular}

${ }^{\star}$ Named species with the most similar 16S rRNA sequence from RDP II (Cole et al., 2003) listed in order of prevalence among all samples.

X87275, AE002098.2 (60971-62514), X06684, D84020, AE014075, AB072699, AY072740, M88157, Z99108, AF243153, L37592, M26923, AE000513 (84836-86337), AE009951 (1072227-1073728), AF356832, U68447, M11656, AB054671, AJ496186, AJ309733, AJ308501, M21774, X80614, AJ011510, AF112175, AB006658, AJ231188, AF477988, AJ010951, AF352533, U75602] were aligned using CLUSTAL W (Thompson et al., 1994). The Jukes and Cantor method (Jukes \& Cantor, 1969) was used to calculate pair-wise genetic distances among the aligned sequences from about nt 65 to 450 in the E. coli sequence. Sequences were then clustered together based on their genetic distances using the neighbour-joining algorithm (Saitou \& Nei, 1987). The distance and clustering analyses were performed using PHYLIP (Felsenstein, 1993). Sequences that were not monophyletic with their most similar sequence from RDP II were checked to determine whether they were chimeras by looking at the BLAST results. Only sequences with similarity to different species in different parts of their sequences (chimeras) were removed from the analysis.

A group of clones was considered a phylotype if their 16S rRNA gene sequences were monophyletic and had less than $3 \%$ nucleotide sequence divergence. The median genetic distances (adjusted fraction of sequence differences) between phylotype sequences and their most similar sequence from RDP II were calculated.

\section{RESULTS AND DISCUSSION}

To characterize the microbial diversity found in the normal human vulva, scrape samples from the labia majora and labia minora of four healthy women were analysed. Partial, single-stranded $16 \mathrm{~S}$ rRNA gene sequences of nearly 900 clones were determined (GenBank accession nos DQ975475-DQ976361) and compared with reference sequence data from RDP II. Each sequence was classified based on the similarity of about $400 \mathrm{nt}$ at the $5^{\prime}$ end of the gene to the corresponding sequence from known bacterial species. The frequencies of phylotypes present in each vulvar sample and their median genetic distance from the most similar 16S rRNA sequence in RDP II are listed in Tables 2 and 3.

\section{The microbiota of the human vulva is diverse}

The study of the human vulva by culture-independent methods resulted in the identification of many phylotypes not previously known to exist at this anatomical site. Previous culture-dependent studies of the vulva have shown that micrococci, $\alpha$ - and $\beta$-haemolytic streptococci, lipophilic diphtheroids, non-lipophylic diphtheroids, bacilli, lactobacilli, Gram-negative and Gram-positive rods, and both $S$. aureus and coagulase-negative staphylococci are present (Aly et al., 1979; Elsner \& Maibach, 1990). Sequences with similarity to representatives from each of these groups of organisms were also found in our study, with the notable exception of $S$. aureus. We cannot explain the absence of $S$. aureus in our samples, but suspect that it may be due to the 
Table 3. Phylotypes unique to one location in each woman

Sample designations (W2-W5) coincide with those of Coolen et al. (2005) and Zhou et al. (2004). A, labia minora; B, labia majora; $n$, number of clones analysed.

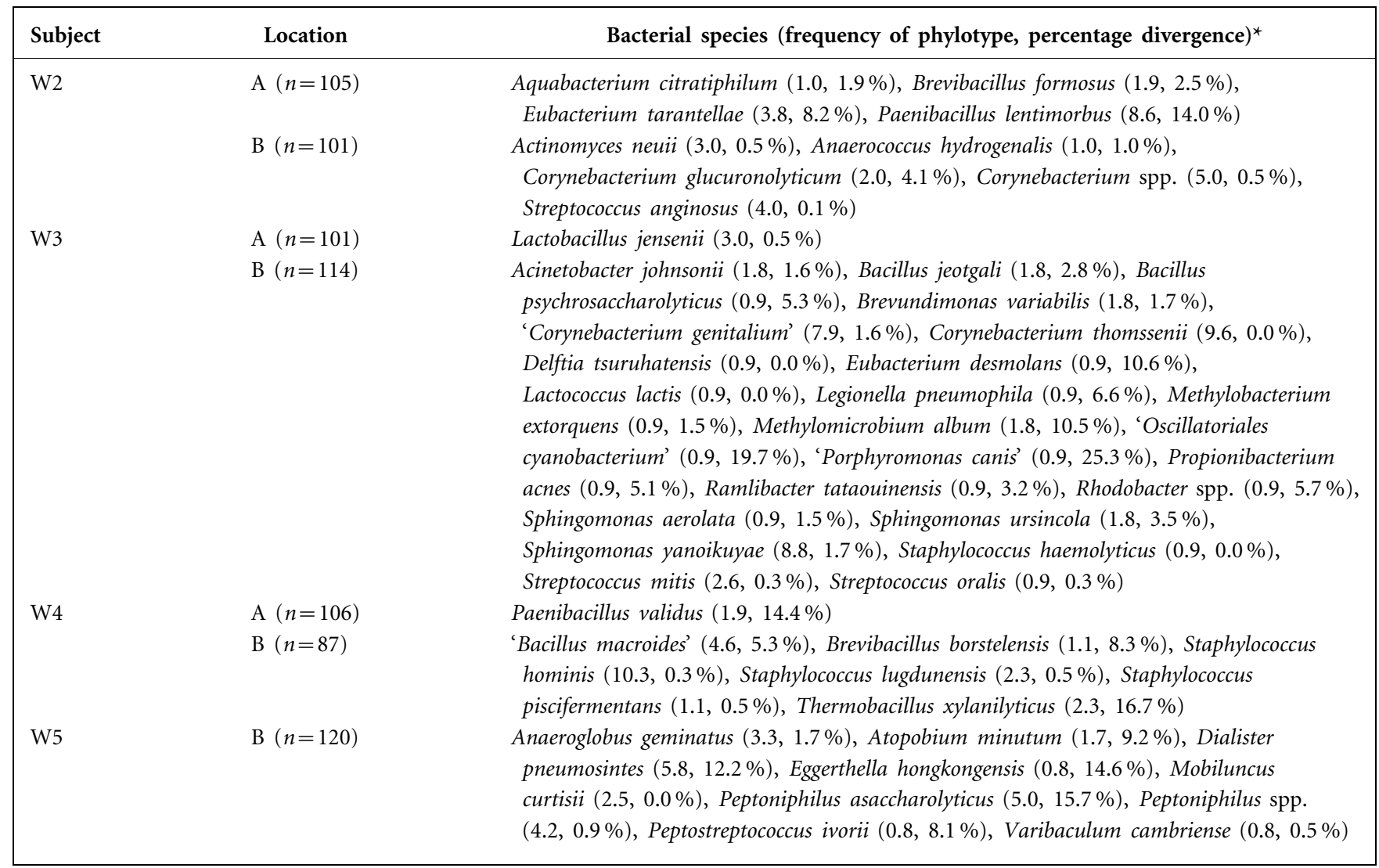

${ }^{*}$ Named species with the most similar 16S rRNA gene sequence from RDP II (Cole et al., 2003). Data are presented as frequency of each phylotype (i.e. percentage of clones examined from each sample identified as that phylotype). The median genetic divergence (base substitutions per 100 bases) of all sequences of that phylotype from the RDP II sequence of the bacterial species are given as a percentage.

use of selective media by previous investigators that allowed them to recover rare populations from their samples. In addition, the sequences of 12 phylotypes diverged by more than $10 \%$ from the closest sequence with a species designation in RDP II, indicating that these twelve phylotypes may belong to previously uncharacterized genera or families of bacteria. Eight phylotypes had $5-10 \%$ sequence divergence from database sequences and may represent new species within known genera.

A total of 64 phylotypes was recovered from samples of the four women, with diverse populations found. The number of microbial populations varied from two phylotypes in the labia minora of W3 to 27 phylotypes from the labia majora of the same woman. Much of the diversity among sites and women was due to the presence of phylotypes that were unique to each individual (Table 3). Most of the unique phylotypes were not abundant; however, some were found in appreciable numbers (5-10\%; Table 3$)$. It should be noted that only numerically abundant populations (those that constituted more than $\sim 1 \%$ of the total community) were represented by the sequences reported, and the possible occurrence of less abundant, yet ecologically important, populations cannot be excluded.

\section{Women have distinctive microbiota}

There were pronounced differences in the composition of vulvar communities of the four women. No two women had the same microbiota and no single phylotype was found in all women. Whilst several phylotypes were detected in more than one woman (Table 2), each woman also had unique phylotypes (Table 3). The most abundant phylotypes in the vulvas of three of the four women were most similar to either Lactobacillus crispatus or Lactobacillus iners, whilst the community of the fourth (W5) was dominated almost equally by phylotypes similar to L. iners, Atopobium vaginae and a phylotype most similar to Megasphaera elsdenii (13-14 \% sequence divergence; Table 2). Differences among women did not appear to relate to either their age or the day 


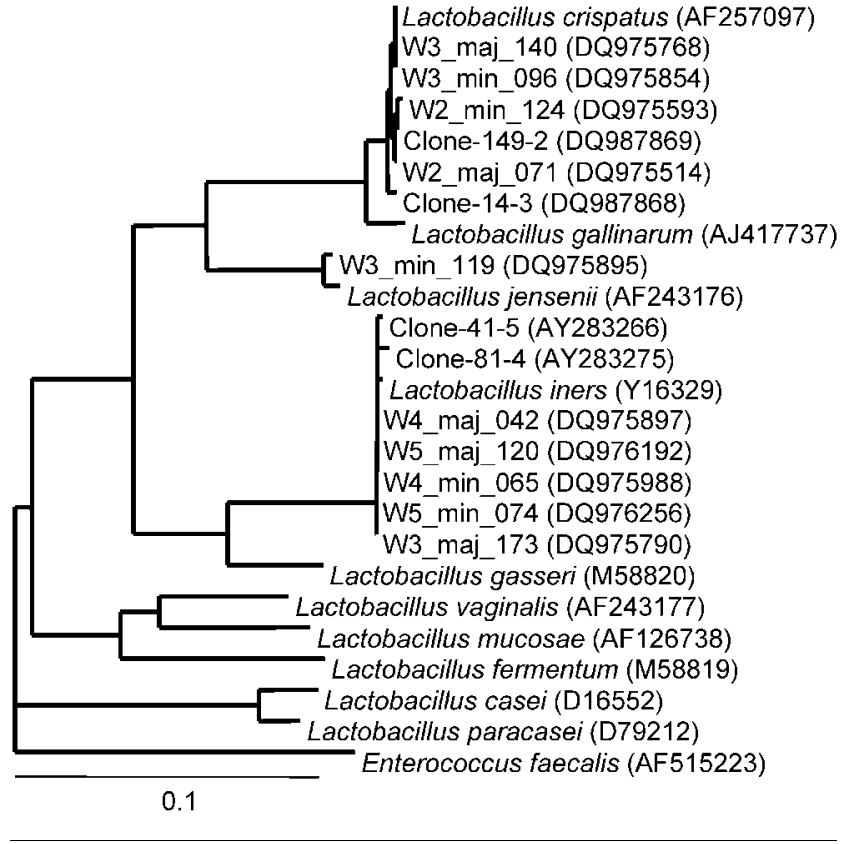

Fig. 1. Phylogenetic relationships among Lactobacillus spp. phylotypes from the labia majora, labia minora and vagina of each woman. Sequences from vulvar samples are labelled Woman_Location_Sequence. Location indicates whether the sequences were from samples of the labia minora ( $\mathrm{min}$ ) or labia majora (maj). Sequences from vaginal samples, labelled clonesequence-woman, are from Zhou et al. (2004). Numbers in parentheses are GenBank accession numbers. Bar, estimated substitutions per nucleotide.

in the menstrual cycle that the samples were taken (Tables 1-3), although the sample size of this study was too small to make conclusive statements.

The vaginal communities of the women in this study were sampled at the same time that the vulvar samples were taken and their composition has been reported previously (Coolen et al., 2005; Zhou et al., 2004). The dominant phylotypes from the vulva were also dominant members of communities in the corresponding vagina. Indeed, the $16 \mathrm{~S}$ rRNA gene sequences of the numerically dominant species in each vulvar sample were almost identical to those in the vagina taken from the same subject at the time (Fig. 1; Zhou et al., 2004). Analysis of these vaginal samples using terminal restriction fragment length polymorphisms have indicated that the vaginal community composition is consistent over the menstrual cycle and over a period of at least 2 months (Coolen et al., 2005).

The vaginal communities of the five women in the study by Zhou et al. (2004) were also commonly encountered among women in a larger study (Forney et al., 2006) that was a subsample of more than 3000 women from across North America (Parsonnet et al., 2005). We expect that a larger study of microbial communities of the vulva will show that vulvar communities are heavily influenced by the vaginal microbiota, and that the communities identified here will reflect healthy vulvar and vaginal communities in general.

\section{Microbiota of the labia majora and labia minora differ and reflect differences in ecotopes}

The microbiota of the two regions of the vulva differed from each other, although the dominant phylotypes from the labia minora were generally dominant members of the labia majora communities of the same subject. Communities of the labia majora were more diverse than those of the labia minora, with 2-14 times as many phylotypes.

The community compositions reflected the different ecotopes of these two regions. As noted above, the microbial community of the labia minora resembled that of the vagina. As was found in culture-dependent studies (Aly et al., 1979; Elsner \& Maibach, 1990), the microbiota of the labia majora included species found on skin, such as Staphylococcus epidermidis and Corynebacterium spp. In addition, we found populations that were probably of faecal origin, such as Enterococcus faecalis.

\section{Conclusions}

These results indicate that the vulva is more complex than originally thought. The small number of participants and single time points suggest that the microbial diversity of the normal human vulva may be even greater than that seen here. It seems likely that various habits and practices of women (such as frequency of bathing, toileting practices and kinds of clothing worn) influence the composition of vulvar communities. Studies that systematically evaluate the effect(s) of such factors are necessary to elucidate the indigenous microbiota of the vulva and identify transient members of these communities.

\section{ACKNOWLEDGEMENTS}

The authors acknowledge Marianne Brunner, R. N., Susan Haines and Deborah C. Schmitz (Procter \& Gamble) for assistance in organizing, conducting and monitoring the clinical study. Audra Johnson is also acknowledged for the program STATGEN, which generated the summary statistics for the pair-wise genetic distances, and for other computational assistance. We also gratefully acknowledge the extraordinary assistance of the editor and two reviewers. The research was supported by NIH grant P20 RR16448 from the COBRE Program of the National Center for Research Resources, NIH grant P20 RR016454 from the INBRE Program of the National Center for Research Resources, and Procter \& Gamble Company, Cincinnati, OH, USA.

\section{REFERENCES}

Altschul, S. F., Madden, T. L., Schaffer, A. A., Zhang, J., Zhang, Z., Miller, W. \& Lipman, D. J. (1997). Gapped BLAST and PSI-BLAST: a new generation of protein database search programs. Nucleic Acids Res 25, 3389-3402.

Aly, R., Britz, M. B. \& Maibach, H. I. (1979). Quantitative microbiology of human vulva. Br J Dermatol 101, 445-448. 
Atassi, F., Brassart, D., Grob, P., Graf, F. \& Servin, A. L. (2006). Vaginal Lactobacillus isolates inhibit uropathogenic Escherichia coli. FEMS Microbiol Lett 257, 132-138.

Berg, R. D. (1999). Bacterial translocation from the gastrointestinal tract. Adv Exp Med Biol 473, 11-30.

Cole, J. R., Chai, B., Marsh, T. L., Farris, R. J., Wang, Q., Kulam, S. A., Chandra, S., McGarrell, D. M., Schmidt, T. M. \& other authors (2003). The Ribosomal Database Project (RDP-II): previewing a new autoaligner that allows regular updates and the new prokaryotic taxonomy. Nucleic Acids Res 31, 442-443.

Coolen, M. J., Post, E., Davis, C. C. \& Forney, L. J. (2005). Characterization of microbial communities found in the human vagina by analysis of terminal restriction fragment length polymorphisms of 16S rRNA genes. Appl Environ Microbiol 71, 8729-8737.

Elsner, P. \& Maibach, H. I. (1990). Microbiology of specialized skin: the vulva. Semin Dermatol 9, 300-304.

Eschenbach, D. A. (2004). Chronic vulvovaginal candidiasis. $N$ Engl $J$ Med 351, 851-852.

Felsenstein, J. (1993). PHYLIP - phylogeny inference package. Department of Genome Sciences, University of Washington, Seattle, USA.

Forney, L. J., Zhou, X. \& Davis, C. C. (2006). Illusions of knowledge about vaginal microbiology. In International Conference on Women and Infectious Diseases: Progress in Science and Action, 16-18 March 2006, p. 48, abstract 11. Atlanta, GA: American Society for Microbiology.

Henderson, B., Wilson, M. \& Hyams, J. (1998). Cellular microbiology: cycling into the millennium. Trends Cell Biol 8, 384-387.

Hooper, L. V., Wong, M. H., Thelin, A., Hansson, L., Falk, P. G. \& Gordon, J. I. (2001). Molecular analysis of commensal host-microbial relationships in the intestine. Science 291, 881-884.

Jukes, T. H. \& Cantor, C. R. (1969). Evolution of protein molecules. In Mammalian Protein Metabolism, pp. 21-132. Edited by $\mathrm{H}$. N. Munro. New York: Academic Press.

Makela, P., Leaman, D. \& Sobel, J. D. (2003). Vulvovaginal trichosporonosis. Infect Dis Obstet Gynecol 11, 131-133.

Merkley, K. (2005). Vulvovaginitis and vaginal discharge in the pediatric patient. J Emerg Nurs 31, 400-402.

Nakatsu, C. H., Korona, R., Lenski, R. E., de Bruijn, F. J., Marsh, T. L. \& Forney, L. J. (1998). Parallel and divergent genotypic evolution in experimental populations of Ralstonia sp. J Bacteriol 180, 4325-4331.

Pace, N. R. (1997). A molecular view of microbial diversity and the biosphere. Science 276, 734-740.

Parsonnet, J., Hansmann, M. A., Delaney, M. L., Modern, A., DuBois, A. M., Wieland-Alter, W., Wissemann, K. W., Wild, J. E., Jones, M. B. \& other authors (2005). Prevalence of toxic shock syndrome toxin 1producing Staphylococcus aureus and the presence of antibodies to this superantigen in menstruating women. J Clin Microbiol 43, 4628-4634.

Rappe, M. S. \& Giovannoni, S. J. (2003). The uncultured microbial majority. Аnnu Rev Microbiol 57, 369-394.

Reid, G. (1999). Urinary tract infection. World J Urol 17, 335-419.

Reid, G. \& Burton, J. (2002). Use of Lactobacillus to prevent infection by pathogenic bacteria. Microbes Infect 4, 319-324.

Saitou, N. \& Nei, M. (1987). The neighbor-joining method: a new method for reconstructing phylogenetic trees. Mol Biol Evol 4, 406-425.

Sheary, B. \& Dayan, L. (2005). Recurrent vulvovaginal candidiasis. Aust Fam Physician 34, 147-150.

Sing, A., Roggenkamp, A., Kress, K., Autenrieth, I. B. \& Heesemann, J. (1998). Bartholinitis due to Streptococcus pneumoniae: case report and review. Clin Infect Dis 27, 1324-1325.

Sobel, J. D. (2001). Antimicrobial resistance in vulvovaginitis. Curr Infect Dis Rep 3, 546-549.

Sobel, J. D. (2003). Erosive vulvovaginitis. Curr Infect Dis Rep 5, 494-498.

Sobel, J. D. (2004). Current trends and challenges in candidiasis. Oncology 18 (Suppl. 13), 7-8.

Spence, D. (2003). Candidiasis (vulvovaginal). Clin Evid 10, 2044 2057.

Tanaka, K., Mikamo, H., Ninomiya, M., Tamaya, T., Izumi, K., Ito, K., Yamaoka, K. \& Watanabe, K. (2005). Microbiology of Bartholin's gland abscess in Japan. J Clin Microbiol 43, 4258-4261.

Thompson, J. D., Higgins, D. G. \& Gibson, T. J. (1994). CLUSTAL W: improving the sensitivity of progressive multiple sequence alignment through sequence weighting, position-specific gap penalties and weight matrix choice. Nucleic Acids Res 22, 4673-4680.

Tlaskalová-Hogenová, H., Stepánková, R., Hudcovic, T., Tucková, L., Cukrowska, B., Lodinová-Zádníková, R., Kozáková, H., Rossmann, P., Bártová, J. \& other authors (2004). Commensal bacteria (normal microflora), mucosal immunity and chronic inflammatory and autoimmune diseases. Immunol Lett 93, 97-108.

Weisburg, W. G., Barns, S. M., Pelletier, D. A. \& Lane, D. J. (1991). $16 \mathrm{~S}$ ribosomal DNA amplification for phylogenetic study. J Bacteriol 173, 697-703.

Wilson, C. (2005). Recurrent vulvovaginitis candidiasis: an overview of traditional and alternative therapies. Adv Nurse Pract 13, $24-29$.

Xu, J. \& Sobel, J. D. (2004). Candida vulvovaginitis in pregnancy. Curr Infect Dis Rep 6, 445-449.

Zhou, X., Bent, S. J., Schneider, M. G., Davis, C. C., Islam, M. R. \& Forney, L. J. (2004). Characterization of vaginal microbial communities in adult healthy women using cultivation-independent methods. Microbiology 150, 2565-2573. 